\title{
Diagnostic yield of endobronchial ultrasound-guided transbronchial needle aspiration for mediastinal staging in lung cancer*
}

\author{
Rendimiento diagnóstico de la ultrasonografía endobronquial con \\ aspiración transbronquial por aguja fina en el estudio de etapificación \\ mediastínica en pacientes con cáncer pulmonar
Sebastián Fernández-Bussy ${ }^{1}$, Gonzalo Labarca ${ }^{2}$, Sofia Canals ${ }^{3}$, lván Caviedes ${ }^{4}$, Erik Folch ${ }^{5}$, Adnan Majid $^{6}$

\begin{abstract}
Objective: Endobronchial ultrasound-guided transbronchial needle aspiration (EBUS-TBNA) is a minimally invasive diagnostic test with a high diagnostic yield for suspicious central pulmonary lesions and for mediastinal lymph node staging. The main objective of this study was to describe the diagnostic yield of EBUS-TBNA for mediastinal lymph node staging in patients with suspected lung cancer. Methods: Prospective study of patients undergoing EBUS-TBNA for diagnosis. Patients $\geq 18$ years of age were recruited between July of 2010 and August of 2013. We recorded demographic variables, radiological characteristics provided by axial CT of the chest, location of the lesion in the mediastinum as per the International Association for the Study of Lung Cancer classification, and definitive diagnostic result (EBUS with a diagnostic biopsy or a definitive diagnostic method). Results: Our analysis included 354 biopsies, from 145 patients. Of those 145 patients, 54.48\% were male. The mean age was 63.75 years. The mean lymph node size was $15.03 \mathrm{~mm}$, and 90 lymph nodes were smaller than $10.0 \mathrm{~mm}$. The EBUS-TBNA method showed a sensitivity of $91.17 \%$, a specificity of $100.0 \%$, and a negative predictive value of $92.9 \%$. The most common histological diagnosis was adenocarcinoma. Conclusions: EBUS-TBNA is a diagnostic tool that yields satisfactory results in the staging of neoplastic mediastinal lesions.
\end{abstract}

Keywords: Lung neoplasms; Bronchoscopy; Endosonography; Neoplasm staging.

\section{Introduction}

Early diagnosis and staging of pulmonary lesions are central to the treatment and survival of patients with suspected lung cancer, especially of those with suspected non-small cell lung cancer. ${ }^{(1)}$ Among the available alternatives, surgery by mediastinoscopy, video-assisted thoracoscopy, or other techniques remains the reference standard for diagnosis and mediastinal staging. However, assessment by minimally invasive techniques, such as flexible bronchoscopy with bronchial or transbronchial biopsy, endobronchial ultrasound (EBUS) with transbronchial needle aspiration (TBNA), virtual navigation, and electromagnetic navigation bronchoscopy, has made it possible to obtain representative histologic and cytologic samples with greater speed and a lower rate of complications. ${ }^{(1,2)}$

Linear EBUS involves the use of a bronchoscope with a convex ultrasound transducer at its distal

\footnotetext{
1. Physician. Division of Interventional Pulmonology, Clinica Alemana of Santiago, Universidad del Desarrollo, Santiago, Chile. 2. Resident in Internal Medicine. Pontifical Catholic University of Chile School of Medicine, Santiago, Chile.

3. Physician. Clinica Alemana of Santiago, Universidad del Desarrollo, Santiago, Chile.

4. Physician. Division of Pulmonology, Clinica Alemana of Santiago, Universidad del Desarrollo, Santiago, Chile.

5. Staff Physician. Division of Thoracic Surgery and Interventional Pulmonology, Beth lsrael Deaconess Medical Center, Harvard Medical School, Boston, MA, USA.

6. Director. Division of Thoracic Surgery and Interventional Pulmonology, Beth lsrael Deaconess Medical Center, Harvard Medical School, Boston, MA, USA.

*Study carried out at the Clinica Alemana of Santiago, Universidad del Desarrollo, Santiago, Chile.

Correspondence to: Sebastián Fernández-Bussy. Avenida Manquehue Norte, 1410, Vitacura, Santiago, Chile.

Tel. 562 2210-1111. Fax: 562 575-4972. E-mail: sfernandezbussy@alemana.cl

Financial support: None.

Submitted: 31 October 2014. Accepted, after review: 20 April 2015.
} 
end to confirm the location of the lesion in real time, which allows fine-needle aspiration of the lesion contents (i.e., TBNA), significantly increasing the diagnostic yield. This method was first used in early 2000 by Herth et al. ${ }^{(3,4)}$

The diagnostic yield of EBUS-TBNA has been studied in different types of airway lesions, as well as in central and peripheral lesions. The procedure has proven to be useful in lesions adjacent to central airway structures, reportedly yielding a sensitivity of 90\% and a specificity of $100 \%$. A systematic review and meta-analysis, ${ }^{(5)}$ which included 11 studies involving 1,299 patients referred for EBUS for mediastinal staging in non-small cell lung cancer, found a sensitivity of 93\% (95\% Cl, 91\%-94\%) and a specificity of 100\% (95\% Cl, 99\%-100\%). However, this diagnostic method is not sufficiently accurate to locate all mediastinal lymph node stations, especially stations $5,6,8$, and 9 of the mediastinal lymph node map developed by the International Association for the Study of Lung Cancer (IASLC). ${ }^{(3,6)}$

The objective of the present study was to describe the diagnostic yield of EBUS-TBNA as a diagnostic and staging method for secondary mediastinal nodal involvement in patients with non-small cell lung cancer.

\section{Methods}

This was a prospective descriptive study of patients undergoing EBUS-TBNA for diagnosis of operable non-small cell lung cancer and referred for mediastinal nodal staging. All procedures performed in outpatients and inpatients of the Clinica Alemana of Santiago, Santiago, Chile, between July of 2010 and August of 2013 were included. Patients $\geq 18$ years of age with a presumptive diagnosis of lung cancer or non-small cell lung cancer who met criteria for surgery were consecutively selected on the basis of clinical history and imaging studies (chest X-ray and CT scans). lung cancer patients with distant metastasis (M1, as per the tumor-node-metastasis classification) $)^{(7)}$ were excluded, as were patients undergoing restaging after chemotherapy and those who refused the procedure.

This study was reviewed and approved by the Research Ethics Committee of the Clinica
Alemana of Santiago, Universidad del Desarrollo, Santiago, Chile.

All procedures were performed with a flexible video bronchoscope (BF-Q180; Olympus Corp., Miami, FL, USA) and a flexible ultrasound bronchoscope (BF-UC180F; Olympus Corp.) by an endoscopist. The procedures were performed in accordance with standard recommendations, with monitoring and sedation under anesthesia. ${ }^{(2)}$ All patients had to sign a written informed consent before undergoing the procedure. The airway and mediastinal lymph node stations were thoroughly inspected with a flexible bronchoscope. Subsequently, the lymph nodes were identified by radiological imaging study and linear transducer ultrasound. Mediastinal lymph nodes with suspected neoplastic involvement-defined as enlargement (10 $\mathrm{mm}$ in size), irregular borders, and irregular shape-that was not confirmed by imaging was aspirated at least six times using a transbronchial needle.

We recorded demographic variables such as age and gender, as well as lesion characteristics on axial CT of the chest, classifying them in accordance with the affected lymph node; location of the lesion in the mediastinum as per the IASLC lymph node map ${ }^{(6)}$; lymph node morphology (round, oval, triangular, or other); lesion borders (defined or irregular); lesion size; and complications associated with the procedure. The anatomopathological study of the samples was carried out at the Department of Pathology by an operator who was blinded to the clinical history and to the diagnostic result of the previous procedure. A sample that was positive for lung cancer was considered to be diagnostic, dispensing with another surgical biopsy. In cases in which bronchoscopy was nondiagnostic, the definitive diagnostic procedure that is defined as the reference standard (surgery by videoassisted thoracoscopy or by mediastinoscopy) was resorted to within 2 months, whereas in cases in which the lesion sample was nondiagnostic by all (bronchoscopic or surgical) methods, a 12-month or longer, chest CT follow-up was defined as the reference standard.

Data were recorded in a database designed in Microsoft Office Excel 2010. The working definitions used for this study were as follows: 
true positive $(\mathrm{TP})=$ an EBUS-TBNA sample positive for lung cancer; true negative $(\mathrm{TN})=$ an EBUS-TBNA sample negative for lung cancer and a surgical sample negative for lung cancer, or axial chest CT follow-up showing no significant changes; false negative $(\mathrm{FN})=$ an EBUS-TBNA sample negative for lung cancer and a surgical sample positive for lung cancer.

In addition, diagnostic yield was defined as the sum of EBUS-TBNA results that were diagnostically positive for cancer and EBUSTBNA results that were negative for cancer and were confirmed by the reference standard $(\mathrm{TP}+\mathrm{TN})$.

On the basis of the results obtained, we assessed sensitivity-defined as TP/(TP + FN)specificity-defined as TN/(TN + FP)-positive predictive value-defined as TP/(TP + FP)-and negative predictive value-defined as $\mathrm{TN} /(\mathrm{TN}$ + FN). Once the diagnostic yield results were obtained, we calculated the positive and negative likelihood ratios for EBUS-TBNA.

\section{Results}

Our analysis included 354 mediastinal lymph node biopsies, from 145 consecutive patients. Of those 145 patients, $54.48 \%$ were male. The mean age was 63.75 years (range, 20 to 88 years). The mean lymph node size was $15.03 \mathrm{~mm}$, and 90 lymph nodes were smaller than $10.0 \mathrm{~mm}$. The demographic and lesion characteristics of the patients included in this study are summarized in Table 1.

Lesions were located as follows: $40.96 \%$ in the lower or upper paratracheal region; $27.96 \%$ in the hilar region; $26.55 \%$ in the subcarinal region; and $4.53 \%$ in the interlobar, lobar, and subsegmental regions. Regarding the ultrasound characteristics of the lymph nodes, 35.0\% were oval, $16.6 \%$ were triangular, and $51.41 \%$ had regular borders, whereas the location of the lesion in the mediastinum as per the IASLC lymph node map and biopsy diagnostic yield is summarized in Tables 2 and 3. The EBUS-TBNA method showed a sensitivity of $91.17 \%$, a specificity of $100.0 \%$, a positive predictive value of $100.0 \%$, and a negative predictive value of $92.9 \%$. The likelihood ratio for a positive diagnostic result was 93.9, whereas the likelihood ratio for a negative diagnostic result was 0.06 .

Analysis of the diagnostic yield of EBUS according to lesion size revealed that the result of $1 / 90$ biopsies was false negative in those lesions smaller than $10 \mathrm{~mm}$, with sensitivity being $90.0 \%$ and specificity being $100.0 \%$ for this group, whereas, in those lesions greater than $10 \mathrm{~mm}$, the yield was $91.25 \%$.

A definitive diagnosis was established by EBUSTBNA in 164 cases, by surgical techniques in 15 cases, and by radiological follow-up in 175 cases. Of the EBUS-TBNA samples that were negative, 15 corresponded to false negatives (pulmonary adenocarcinoma, in 11; squamous neoplasia, in 4).

Of all pulmonary lesions that were diagnosed by any method, 170 were found to be consistent with lung cancer, whereas 9 were of benign etiology. The prevalence of lung cancer in our series was $48.02 \%$. The histologic results are summarized in Table 4.

Finally, in our series, there was one episode of pneumomediastinum that was considered related to the procedure and occurred after biopsy sampling of the pretracheal region, which was performed as part of mediastinal lymph node assessment. Management of this complication was medical, without major complications, with a 2-day hospital stay.

\section{Discussion}

Assessment of pulmonary lesions by minimally invasive techniques plays an increasingly important

Table 1 - Demographic characteristics of and lymph node size in the patients included in the study ( $\mathrm{N}$ $=145$ ).

\begin{tabular}{lcc}
\hline \multicolumn{1}{c}{ Characteristic } & $\mathrm{n}$ & $\%$ \\
\hline Females & 66 & 45.51 \\
Males & 79 & 54.48 \\
Mean age, years & 63,75 & $20-88^{\mathrm{a}}$ \\
Total number of biopsies & 354 & \\
Size, mm & & \\
$\quad<5$ & 6 & 1.64 \\
$5-9$ & 84 & 22.75 \\
$10-14$ & 157 & 41.39 \\
$15-19$ & 50 & 17.01 \\
$>20$ & 57 & 17.21 \\
\hline
\end{tabular}

aRange. 
Table 2 - Location of the lymph nodes in the mediastinum as per the IASLC classification and biopsy results.

\begin{tabular}{ccccc}
\hline Location & Samples & Positive biopsies & Negative biopsies & False-negative biopsies \\
\hline 1 & 1 & 1 & 0 & 0 \\
$2 \mathrm{~L}$ & 3 & 2 & 1 & 0 \\
2R & 11 & 7 & 4 & 1 \\
$4 \mathrm{~L}$ & 45 & 21 & 24 & 0 \\
$4 \mathrm{R}$ & 85 & 37 & 48 & 6 \\
7 & 94 & 44 & 50 & 5 \\
$10 \mathrm{~L}$ & 45 & 15 & 30 & 1 \\
$10 \mathrm{R}$ & 54 & 17 & 37 & 0 \\
$11 \mathrm{R}$ & 11 & 9 & 2 & 0 \\
$11 \mathrm{~L}$ & 2 & 0 & 2 & 0 \\
$12 \mathrm{R}$ & 3 & 2 & 1 & 15 \\
Total & 354 & 155 & 199 & 0 \\
\hline
\end{tabular}

IASLC: International Association for the Study of Lung Cancer.

Table 3 - Ultrasound characteristics of the lymph nodes aspirated $(\mathrm{N}=354)$ under EBUS guidance for mediastinal staging.

\begin{tabular}{lcc}
\hline \multicolumn{1}{c}{ Characteristic } & $\mathrm{n}$ & $\%$ \\
\hline Shape & & \\
Oval & 124 & 35.02 \\
Round & 148 & 41.8 \\
Triangular & 59 & 16.66 \\
$\quad$ Other & 23 & 6.49 \\
Borders & & \\
$\quad$ Regular & 182 & 51.41 \\
$\quad$ lrregular & 172 & 48.58 \\
\hline
\end{tabular}

EBUS: endobronchial ultrasound.

Table 4 - Definitive histologic results for the EBUSTBNA samples.

\begin{tabular}{lc}
\hline \multicolumn{1}{c}{ Histologic result } & $\%$ \\
\hline Neoplasia & $(\mathrm{n}=170)$ \\
Adenocarcinoma & 69.41 \\
Squamous disease & 18.82 \\
Metastasis & 1.76 \\
Small cell disease & 7.64 \\
Neuroendocrine disease & 2.35 \\
Non-Hodgkin's lymphoma & - \\
Benign lesion & $(\mathrm{n}=9)$ \\
Sarcoidosis granuloma & 4.70 \\
Tuberculosis granuloma & 11.1 \\
\hline
\end{tabular}

EBUS-TBNA: endobronchial ultrasound-guided transbronchial needle aspiration.

role as diagnostic yields are increased. EBUS has been used as a method that allows TBNA of pulmonary lesions to be performed reliably and safely. Methodologically, studies analyzing the diagnostic yield of pulmonary lesions suggestive of cancer do not have the reference standard applied to all positive histologic results. The main reason for this is that it is complicated to perform another diagnostic surgical procedure in patients diagnosed with lung cancer.

Another application of EBUS-TBNA is its ability to stage lung cancer in the mediastinum, mainly because the technique allows easy access to most lymph node stations, except for stations 5, 6, 8, and 9 (IASLC classification), from which it is not possible to obtain histologic or cytologic samples by using this technique; in such cases, EBUS-TBNA can be complemented with ultrasound-guided endoscopy. ${ }^{(8)}$ However, the yield of EBUS-TBNA is significantly decreased in peripherally located pulmonary lesions..$^{(3,8)}$

The diagnostic yield of mediastinal staging by EBUS-TBNA is high, with a reported sensitivity of approximately $90 \%$ and a specificity of $100 \%$. In a recently published randomized clinical trial, ${ }^{(9)}$ the yield of EBUS-TBNA as the first-choice minimally invasive method for the staging and diagnosis of lung cancer in lesions adjacent to the central airways was compared with that of commonly used techniques. The group randomized to EBUS-TBNA had a decrease in the number of unnecessary thoracotomies and complications, as well as a decrease in the time required to confirm or rule out the diagnosis of lung cancer and make therapeutic decisions. In that study, EBUS-TBNA had a sensitivity of $92 \%$ and a specificity of $100 \%$. 
In our series, the diagnostic yield of EBUSTBNA was high, especially because all lesions were located in the central airway, with lesions visualized by EBUS. We evaluated the yield of EBUS-TBNA and found that, in small lesions (those smaller than $10 \mathrm{~mm}$ ), it remained satisfactory. This finding is a contribution to the assessment of solitary pulmonary nodules and lymph nodes between 5 and $10 \mathrm{~mm}$, given that lesions smaller than $7 \mathrm{~mm}$ are not seen on imaging studies such as positron emission tomography-CT, whereas, by using ultrasound, those lesions can be found and aspirated transbronchially, with a diagnostic yield of $90 \%$ or more.

Tedde et al., ${ }^{(10)}$ the only available reference regarding EBUS-TBNA in South America, included 50 patients who underwent a total of 201 EBUSTBNA biopsies of 81 lymph nodes or mediastinal masses for diagnosis and staging. In that series, the diagnosis of cancer was confirmed in 57\% of the patients in whom EBUS-TBNA was diagnostic. A negative point in that study was that, in 13 of the 50 patients, the cytologic material was not optimal for anatomopathological examination.

Complications associated with EBUS-TBNA are rare and usually minor. In our series, there was one case of pneumomediastinum-a complication with a reported rate of less than 1\%-that was managed medically, without other major complications. In the literature, complications are infrequent, with self-limited bleeding being reported in less than 5\% of the procedures, whereas the rate of pneumothorax is $1 \%$ with no fatal events, ${ }^{(8,11)}$ similar to what was found in our series.

This study has some limitations. First, the study design: the protocol for studies of diagnostic yield should be in accordance with the recommendations of the STARD statement. ${ }^{(12)}$ In our study, those diagnostic procedures that were positive for lung cancer by histology were not confirmed by surgical biopsy or surgical histology (the gold standard), and therefore it is not possible to determine the proportion of false positives. However, given that a positive histologic result for cancer is confirmatory, it should not be a confounding variable. Second, the prevalence found in our study was lower than that reported in other series of lung cancer patients, in which prevalence rates range from $70 \%$ to $75 \%$. Third, this study was conducted in a single center, with the same operator. Diagnostic yield is a learning curve-dependent variable, and this can reduce result applicability to other centers with less experience in EBUS-TBNA.

In conclusion, EBUS-TBNA is a diagnostic tool with a high diagnostic yield, has few associated complications, and should be considered an option for mediastinal nodal staging in patients with lung cancer, especially in those with non-small cell lung cancer.

\section{References}

1. Rivera MP, Mehta AC, Wahidi MM. Establishing the diagnosis of lung cancer: Diagnosis and management of lung cancer, 3rd ed: American College of Chest Physicians evidence-based clinical practice guidelines. Chest. 2013;143(5 Suppl):e142S-65S.

2. Ernst A, Silvestri GA, Johnstone D; American College of Chest Physicians. Interventional pulmonary procedures: Guidelines from the American College of Chest Physicians. Chest. 2003;123(5):1693-717. http://dx.doi.org/10.1378/ chest.123.5.1693

3. Anantham D, Koh MS, Ernst A. Endobronchial ultrasound. Respir Med. 2009;103(10):1406-14. http://dx.doi. org/10.1016/j.rmed.2009.04.010

4. Dincer HE. Linear EBUS in staging non-small cell lung cancer and diagnosing benign diseases. J Bronchology Interv Pulmonol. 2013;20(1):66-76. http://dx.doi. org/10.1097/LBR.0b013e31827d1514

5. Gu P, Zhao YZ, Jiang LY, Zhang W, Xin Y, Han BH. Endobronchial ultrasound-guided transbronchial needle aspiration for staging of lung cancer: a systematic review and meta-analysis. Eur J Cancer. 2009;45(8):1389-96. http://dx.doi.org/10.1016/j.ejca.2008.11.043

6. Rusch VW, Asamura H, Watanabe H, Giroux DJ, RamiPorta R, Goldstraw P, et al. The IASLC lung cancer staging project: a proposal for a new international lymph node map in the forthcoming seventh edition of the TNM classification for lung cancer. $\mathrm{J}$ Thorac Oncol. 2009;4(5):568-77. http://dx.doi.org/10.1097/ JT0.0b013e3181a0d82e

7. Tanoue LT, Detterbeck FC. New TNM classification for non-small-cell lung cancer. Expert Rev Anticancer Ther. 2009;9(4):413-23. http://dx.doi.org/10.1586/era.09.11

8. Vilmann P, Puri R. The complete "medical" mediastinoscopy (EUS-FNA + EBUS-TBNA). Minerva Med. 2007;98(4):331-8.

9. Navani N, Nankivell M, Lawrence DR, Lock S, Makker $\mathrm{H}$, Baldwin DR, et al.; lung-B00ST trial investigators. Lung cancer diagnosis and staging with endobronchial ultrasound-guided transbronchial needle aspiration compared with conventional approaches: an openlabel, pragmatic, randomised controlled trial. Lancet Respir Med. 2015;3(4):282-9. http://dx.doi.org/10.1016/ S2213-2600(15)00029-6

10. Tedde ML, Figueiredo VR, Terra RM, Minamoto $\mathrm{H}$, Jatene FB. Endobronchial ultrasound-guided transbronchial needle aspiration in the diagnosis and staging of mediastinal lymphadenopathy: initial experience in Brazil. J Bras Pneumol. 2012;38(1):33-40. 
11. von Bartheld MB, van Breda A, Annema JT. Complication rate of endosonography (endobronchial and endoscopic ultrasound): a systematic review. Respiration. 2014;87(4):343-51. http://dx.doi. org/10.1159/000357066
12. Bossuyt PM, Reitsma JB, Bruns DE, Gatsonis CA, Glasziou $\mathrm{PP}$, Irwig LM, et al. The STARD statement for reporting studies of diagnostic accuracy: explanation and elaboration. Ann Intern Med. 2003;138(1):W1-12. http://dx.doi. org/10.7326/0003-4819-138-1-200301070-00010 